\title{
Spectre, mort vivant et autre figure fatale dans trois nouvelles de Hanns Heinz Ewers
}

Gespenster, Wiedergänger und andere fatale Figuren in drei Novellen von Hanns Heinz Ewers

Phantom, zombie and other fatal characters in three short stories from Hanns

Heinz Ewers

\section{Alain Cozic}

\section{(2) OpenEdition} Journals

\section{Édition électronique}

URL : https://journals.openedition.org/ceg/10788

DOI : $10.4000 /$ ceg. 10788

ISSN : 2605-8359

\section{Éditeur}

Presses Universitaires de Provence

\section{Édition imprimée}

Date de publication : 1 octobre 2012

Pagination : 335-354

ISBN : 0751-4239

ISSN : 0751-4239

Référence électronique

Alain Cozic, "Spectre, mort vivant et autre figure fatale dans trois nouvelles de Hanns Heinz Ewers », Cahiers d'Études Germaniques [En ligne], 63 | 2012, mis en ligne le 07 septembre 2020, consulté le 05 juin 2021. URL : http://journals.openedition.org/ceg/10788 ; DOI : https://doi.org/10.4000/ceg.10788 


\title{
Spectre, mort vivant et autre figure fatale dans trois nouvelles de Hanns Heinz Ewers
}

\author{
Alain COZIC \\ Université de Toulouse 2 - Le Mirail
}

\begin{abstract}
Atome, fantômes
Né en 1871, Hanns Heinz Ewers fait paraître en 1907 et 1909 deux recueils de nouvelles, Das Grauen et Die Besessenen, qui marquent une étape décisive dans sa jeune carrière littéraire, inscrivant désormais "l'épouvante" au cœur de ses préoccupations et de son écriture. Très tôt, Ewers s'est intéressé au spiritisme, aux sciences occultes et à l'hypnose en particulier ; il a lu Baudelaire, Gautier - dont il traduira les œuvres avec sa femme -, Maupassant, Verlaine, Maeterlinck, d'Annunzio, Oscar Wilde. Ses modèles de l'époque sont avant tout Heine, Poe et Hoffmann. Sur le grand auteur fantastique américain, il écrit un essai en 1905, Edgar Alan Poe, où, songeant à celui qu'il admire et lui adjoignant les noms d'E. T. A. Hoffmann, JeanPaul, Villiers de 1'Isle-Adam et Baudelaire, il propose une définition de "l'artiste", en l'occurrence de l'écrivain, qui, à l'évidence, pourrait s'appliquer aussi à lui-même ${ }^{1}$. On remarquera que, lorsqu'il évoque les sujets abordés par Poe dans ses "histoires", c'est pour souligner aussi que jusqu'à présent "la science" a été impuissante à percer ces mystères, si tant est qu'elle ait été au fait de leur présence. Ewers n'aura désormais de cesse qu'il n'ait parcouru lui aussi semblables territoires obscurs, "terres inexplorées" ("Neuland") qui restent en effet à défricher et à déchiffrer.

Trois nouvelles seront ici prises en compte, la plus célèbre d'Ewers sans doute, Die Spinne (L'Araignée), et deux autres, Der tote Jude (Le Juif mort)

\footnotetext{
Wie wenige verdienen in diesem heiligen Sinne den stolzen Namen! Th. A. Hoffmann [sic]
}

1 "Was ist — im engsten, im besten Sinne — der Künstler? E i n P i o n i e r d e r K u 1 t u r ind a s e u l and des Un bew ussten! verdient ihn, und Jean Paul und Villiers und Baudelaire - Und ganz sicher auch Edgar Allan Poe, $[\ldots]$ der in so manchen seiner Geschichten ein geheimes Land der Seele betrat, von dem niemand vor ihm - und am wenigsten die Wissenschaft - eine leise Ahnung hatte!": Hanns Heinz Ewers, Edgar Allan Poe, Berlin und Leipzig, Schuster und Loeffler, 1905, p. 18-19. C'est Ewers lui-même qui souligne.
\end{abstract}


et Der letzte Wille der Stanislawa d'Asp (Les dernières volontés de Stanislawa d'Asp), qui ont moins retenu l'attention ${ }^{2}$. L'approche choisie consistera à analyser le "phénomène" qui entre en scène dans chacun de ces trois textes, "l'inquiétante", sinon mortelle "étrangeté" se manifestant à un moment donné dans l'existence des personnages témoins, voire victimes de ce surgissement brutal, dans leur quotidien, de l'inconcevable. Publiant ses récits à l'orée du $\mathrm{XX}^{\mathrm{e}}$ siècle, Ewers, par les thématiques qu'il y aborde et les modalités d'écriture qu'il met en œuvre, s'inscrit parfaitement dans une tradition européenne bien établie au cours du siècle précédent, tradition qu'il paraît même porter à un réel point d'incandescence ${ }^{3}$.

Durant les trois décennies qui séparent la naissance d'Ewers de la publication de ces deux volumes de nouvelles s'achève progressivement le $\mathrm{XIX}^{\mathrm{e}}$ siècle. Période charnière - "Jahrhundertende" et "Jahrhundertwende" pourrait-on dire en allemand - que les termes de "fin de siècle", de "décadence", de "décadentisme" notamment s'efforcent, selon les cas et en fonction de leurs utilisateurs, de définir. Même si l'on associe l'idée de décadence à cette atmosphère de "fin de siècle" précisément, sans doute peuton en percevoir les germes dès 1850 chez Baudelaire, la voir s'affirmer en France dans les années 1870 où la guerre, les événements douloureux de la Commune ont pu faire naître chez les écrivains et les artistes de l'époque le sentiment qu'un monde disparaissait, que s'évanouissait une civilisation. En publiant ses Essais de psychologie contemporaine près de quarante ans après Baudelaire, en 1883, Paul Bourget analyse chez des auteurs comme ce même Baudelaire, Taine, Renan ou Stendhal, ce qu'il définit comme une véritable maladie, faite d'inquiétude, de nervosité, de mélancolie, de pessimisme. Peur, insatisfaction de vivre, mal être, déréliction qu'incarne - quintessenciés - Des

2 "Die Spinne", "Der letzte Wille der Stanislawa d'Asp", in: Die Besessenen. Edition utilisée pour la présente étude : Hans Heinz EwERS, Die Besessenen, München, Georg Müller, 1925. Edition désormais identifiée dans les notes de bas de page par $D B$, suivi du numéro des pages.

"Der tote Jude", in : Das Grauen. Seltsame Geschichten. Edition utilisée pour la présente étude: Hans Heinz Ewers, Das Grauen, München, Georg Müller, 1920. Edition désormais identifiée dans es notes de bas de page par $D G$, suivi du numéro des pages.

3 Cette étude se situe dans le prolongement de celles - décisives - menées par Jean-Jacques POLLET sur Ewers. Conformément à l'esprit du présent volume, elle veut mettre surtout l'accent sur la subversion du réel produite chaque fois qu'entrent en jeu chez Ewers spectres et autres créatures maléfiques.

De Jean-Jacques POLLET, voir en particulier :

- Essai sur la littérature fantastique allemande au début du XX siècle (1900-1930). Thèse de doctorat d'Etat. 1985.

- La fiancée du diable. Nouvelles fantastiques allemandes de 1900. Réunies et présentées par Jean-Jacques Pollet. Textes traduits par J.-J. POLLET, P. GIRAUD, G. RICCARDI. Paris, Albin Michel, 1994.

- Introduction à la nouvelle fantastique allemande, Paris, Nathan, 2007.

- Ecritures fantastiques allemandes, Artois Presses Universités, 2010. 
Esseintes, le héros de $A$ rebours de Joris-Karl Huysmans, publié un an plus tard en 1884.

Le XIX $^{\mathrm{e}}$ siècle qui s'achève fut aussi, et notamment dans sa seconde partie, celui du progrès scientifique et technologique, de son développement, exponentiel et fulgurant, dans de multiples domaines. Qu'on en juge par quelques repères.

Invention de la pile électrique (Volta), du métier à tisser (Jacquard), de la presse à imprimer mécanique (Koenig), du stéthoscope (Laennec), du téléphone (Bell), du moteur à quatre temps (Reithmann), naissance de la photographie (Daguerre), du cinématographe (les frères Lumière); découverte des lois de la dilatation des gaz (Gay-Lussac), des lois fondamentales de l'électromagnétisme (Ampère), de celles du courant électrique $(\mathrm{Ohm})$, du noyau cellulaire (Brown), de la photosynthèse (von Sachs), des chromosomes (Flemming), du vaccin antirabique (Pasteur), du bacille de la peste (Yersin), des rayons X (Röntgen), du radium (Pierre et Marie Curie), du rayonnement des corps radioactifs (Becquerel). Champollion déchiffre les hiéroglyphes, Morse crée la première ligne télégraphique, l'homme de Neandertal puis celui de Cro-Magnon sont découverts, les premiers puits de pétrole sont exploités aux USA, le canal de Suez est percé, le métro de Londres mis en service, Mendeleïev propose sa classification des éléments chimiques, Taylor met au point son organisation du travail, Clément Ader et sa machine volante décollent sur deux cents mètres, les premiers gratte-ciels sont construits à Chicago, on traverse l'Atlantique sur des navires désormais à vapeur, Charcot étudie les centres fonctionnels du cerveau, Claude Bernard fonde la physiologie, la première ligne de chemin de fer circule en Angleterre, le premier câble transatlantique est posé, les frères Hyatt fabriquent le celluloïd, première matière artificielle, Hertz découvre les ondes électromagnétiques et Nobel la dynamite, Panhard et Levassor fabriquent la première automobile à essence, l'aspirine est produite industriellement. En 1905 - pour envisager le changement de siècle -, Einstein fait connaître sa théorie de la relativité, rendant caduque la physique newtonienne, rupture épistémologique s'il en est, en 1900, Freud publie L'interprétation des rêves et Cinq leçons sur la psychanalyse en 1909, en 1913, Bohr et Rutherford découvrent la structure de l'atome.

L'homme fait donc sien le monde, sur et sous terre, sur mer, dans les airs, il commence à percer les secrets de l'atome comme ceux du cerveau, il fabrique en masse et en série, il se déplace, de plus en plus loin et de plus en plus vite, il communique toujours plus rapidement, il met au point des traitements contre les maladies, il sonde son passé et son histoire, s'approprie le présent, prépare l'avenir, il découvre, invente, innove, sans relâche. Avancées scientifiques sans commune mesure, sans équivalent par rapport au siècle précédent, qui entraînent autant de bouleversements dans l'évolution des idées sociales et philosophiques, des conceptions du monde, des rapports humains. Ce siècle est aussi celui de la massification que produit la révolution 
industrielle, du capitalisme naissant, de la prolétarisation d'une partie de la société, de la paupérisation croissante de certaines de ses catégories, des luttes sociales, des doctrines qui s'opposent. Il est celui de la mort de Dieu tout autant que celui de la recherche de l'origine de l'homme. Feuerbach publie L'Essence du christianisme en 1841, Darwin De l'origine des espèces par voie de sélection naturelle en 1859, Marx le premier livre du Capital en 1867, Nietzsche écrit son œuvre entre 1871 et 1887.

Siècle de la science, le $\mathrm{XIX}^{\mathrm{e}}$ siècle érige l'approche scientifique en modèle. L'influence de la pensée positiviste d'Auguste Comte qui publie son Cours de philosophie positive entre 1830 et 1842 est considérable. Le positivisme, "l'esprit scientifique" deviennent des références absolues. Et, en effet, comment pourrait-on remettre en question les principes qui les fondent, comment renier cette appropriation du monde fondé sur l'observation des faits et l'analyse des lois qui les régissent, comment dédaigner la raison, raisonnable et raisonnante, outil jusqu'alors inégalé pour saisir et comprendre la réalité ?

Et pourtant, cet édifice rationaliste dense qui paraît inexpugnable est traversé de fissures. Dans la dernière décennie du siècle, Bergson, qui publie en 1889 Essais sur les données immédiates de la conscience, repense les rapports de l'homme et du monde, ouvre de nouveaux horizons spirituels, fait une méthode de l'intuition comme mode de connaissance directe et immédiat. Tout aussi fondamentalement, et tout au long du siècle, en contrepoint de la science, du règne de la raison, surgit "l'irrationnel", sous ses multiples aspects. Comme si la science ne pouvait avoir le dernier mot, ni l'esprit humain se laisser enfermer aussi aisément dans des catégories qui permettent par ailleurs de maitriser la matière.

"Le sommeil de la raison engendre des monstres", écrit Goya sur le frontispice de l'un de ses Caprices, quatre-vingts estampes gravées à l'eauforte conçues dès 1799 où se mêlent rêves, fantaisies et réalisme cru. Entre 1821 et 1823 , reclus dans sa maison, il peint quinze toiles qui disent l'absurdité du monde, la cruauté de l'homme, la nature dévastatrice, la présence de la mort, comme une descente aux enfers que Saturne, géant se repaissant d'un corps de femme ou d'adolescent, semble symboliser à lui seul. Füßli, dans ses "cauchemars", laisse libre cours à ses fantasmes, ses visions inquiétantes, son imaginaire débridé. Les fantasmagories de John Martin, en 1817, semblent le reflet des visions provoquées par sa consommation de l'opium, des plus répandues à l'époque. William Blake, séduit par la Divine Comédie de Dante, peint en 1824 Le Cercle de luxure, l'une des scènes les plus célèbres de l'ouvrage, où l'on voit, dans une spirale qui traverse toute la surface du tableau, les corps de ceux que la passion a égarés emportés dans un tourbillon ininterrompu. Le cycle de six gravures sur bois d'Alfred Rethel, en 1849, reprend le thème éternel de la danse des morts et montre la grande faucheuse en personne. Le rêve, l'irréel, le lyrisme

4 "El sueño de la razón produce monstruos". 
visionnaire semblent imprégner la peinture d'Arnold Böcklin, dont L'île des morts, en 1880, avec son atmosphère mystérieuse et inquiétante, est l'une des toiles les plus caractéristiques. Et que dire du monde désenchanté, énigmatique qui paraît renvoyer aux ténèbres de l'âme humaine d'un Edward Munch ? De la vision des plus personnelles de Camille Claudel encore lorsqu'elle sculpte la Parque Clotho, celle qui file le destin des hommes, sous les traits d'une vieillarde, comme si elle voulait signifier par là que, dès la naissance, l'homme est condamné ?

Scientifique et rationaliste le $\mathrm{XIX}^{\mathrm{e}}$ siècle ? Assurément, mais imprégné aussi d'une "inquiétante étrangeté". Poursuivons. Les Fantasiestücke et Nachtstücke de E.T.A. Hoffmann sont respectivement publiés en 1814 et 1817, Die Elixiere des Teufels en 1815-1816, Die Serapionsbrüder en 18191821. Poe - le maître - écrit l'essentiel de son œuvre entre 1840 et 1845. Ambrose Bierce, son compatriote, publie son Dictionnaire du diable et ses Fables fantastiques en 1906, Nodier Smarra ou les démons de la nuit en 1821, Mérimée La Vénus d'Ille en 1837, Erckmann-Chatrian leurs Contes fantastiques en 1860, le Fanu, Les Créatures du miroir avec sa nouvelle la plus célèbre, Carminat, en 1872, Barbey d'Aurevilly Les Diaboliques en 1874, Villiers de l'Isle-Adam ses Contes cruels et Nouveaux contes cruels en 1883 et 1888 , Maupassant Le Horla en 1886, Stevenson Le Cas étrange du Dr Jekyll et de Mr Hyde en 1886.

Le $\mathrm{XIX}^{\mathrm{e}}$ siècle ? Röntgen et les rayons $\mathrm{X}$, oui ; mais tout autant le spectre de La femme amoureuse de Villiers de l'Isle-Adam. Les travaux de Charcot et de Claude Bernard en médecine, bien entendu ; la rencontre du Dr Jekyll et de son double, tout aussi évidemment. Le téléphone et le télégraphe, à l'évidence ; les automates et les statues qui s'animent chez Hoffmann et Mérimée, de la même façon.

S'établit ainsi au fil de ces décennies un dispositif fait d'approches différenciées de la réalité, de conceptions différentes du monde et de l'homme, de représentations diverses du réel, de schémas perceptifs variés, qui définit le siècle dans sa pluralité, une épistémé pour le dire comme Foucault.

Ewers dans cette constellation? En son cœur même lorsqu'il naît en 1871, dans son prolongement direct lorsqu'il écrit Das Grauen et Die Besessenen, quand on sait les maîtres es étrangeté dont il se réclame. Ses morts vivants et ses spectres n'ont rien à envier à ceux de Villiers de l'Isle Adam, la morbidité des actions que ses personnages accomplissent rien à celle des figures de Barbey d'Aurevilly, ses femmes fatales sont les égales de celles de Mérimée, quand elles ne les dépassent pas en cruauté. Si le siècle fait mourir Dieu, le Diable, pourrait-on dire, lui survit. Le Malin est coriace en effet, ne s'en 
laisse pas conter, est prompt à produire force avatars, surtout quand la malignité est ancrée au tréfonds des êtres ${ }^{5}$. Ewers le démontre avec maestria.

\section{Le Juif mort : Un rire post-mortem qui rend fou}

Deux personnages sont présents dans les premières lignes de la nouvelle, l'un rappelant à l'autre un certain jour et lui demandant de raconter, le second se montrant dans un premier temps des plus réticents à remémorer des souvenirs manifestement terrifiants, mais finissant par obtempérer tout de même sans se faire beaucoup prier certes. "En outre il n'est pas du tout vrai que vous ne voulez pas raconter, lui affirme péremptoirement son interlocuteur, vous voulez parler et nous devons écouter. Ecoutons donc" Désir de l'un d'entendre de nouveau - à l'évidence, il connaît déjà l'histoire, mais ne se lasse pas de l'écouter - un récit permettant aussi "de ne pas oublier que de nos jours encore le sang coule dans ce meilleur des mondes qui soit"7. Comme une nécessité incoercible chez l'autre de relater encore des faits auxquels il a été mêlé, quatorze ans auparavant, comme nous l'apprenons à la fin.

Par ce dialogue, Ewers crée donc une situation narrative initiale qui ne détonne pas dans ce type de nouvelle : un récit-cadre qui met en scène deux personnages, l'un d'entre eux devenant ensuite le conteur unique du récit enchâssé. Une phrase, dans les dernières lignes de la nouvelle, referme ici le récit enchâssant sans que l'interlocuteur du narrateur intervienne de nouveau. La mise en perspective temporelle, toutefois, est importante. Le récit qu'on lit est nécessairement rétrospectif, relation dans le présent du narrateur d'événements du passé que la mémoire restitue. L'insistance de l'interlocuteur poussant son ami à raconter peut être perçue comme une manière de curiosité perverse, morbide à écouter de nouveau, une fois encore, la relation de faits s'étant déroulés un jour donné ${ }^{8}$, qui sortent à l'évidence de l'ordinaire et sont "d'une nature effrayante". Le "rire" qui accompagne sa demande peut du reste exprimer une forme de distance qu'il sait prendre, qu'il a gagnée par rapport aux événements en question. Malin plaisir de cet auditeur à entendre de nouveau cette histoire, sans qu'elle le traumatise pour autant, suave perspective à l'idée de parcourir les sentiers de l'épouvante dès

5 Sur le diable et ses avatars comme éléments constitutifs de l'imaginaire occidental, voir l'étude de Robert MUCHEMBLED : Une histoire du diable. XII $-X X^{e}$ siècle, Paris, Editions du Seuil, 2000.

6 "Außerdem ist das gar nicht wahr, dass Sie nicht erzählen wollen: Sie wollen sprechen und wir sollen hören. Also hören wir", $D G, 212$.

7 "Es ist gut, dass man nicht vergisst, dass auch heute noch Blut fließt in dieser besten aller Welten", Ibid.

8 "Und nun ist der Tag gekommen, an welchem vor nunmehr —,", DG, 211.

9 'Der andere sagte: 'Nein. — Aber es sind Erinnerungen - '.

'_ - so unerhört erschrecklicher Natur, dass Stein und Bein gefrieren' lachte der Schauspieler", Ibid. 
lors que c'est l'autre, et non lui-même, qui a été contraint de les emprunter un jour.

Le narrateur, quant à lui, s'il faut en croire son auditeur, "veut" raconter, "parler", se rappeler encore ces "souvenirs". "Alors, je vous en prie, soulagez-vous", lui dit même l'autre ${ }^{10}$, semblant prêter ainsi au fait de conter quelque vertu thérapeutique, cathartique. Dire (encore), verbaliser (de nouveau), extérioriser par des mots l'horreur pour (tenter de) la surmonter, la bannir (définitivement?) en somme. Il n'est pas certain que cette vertu supposée que l'auditeur prête au récit conté, aux événements ainsi oralement reconstitués - et devant un témoin attentif - aura sur le narrateur les effets prétendument escomptés. On serait même plutôt tenté d'affirmer le contraire. Ce fameux jour - on comprendra plus tard de quelle journée particulière il s'agit - et les faits qui se sont déroulés semblent être pour le narrateur des souvenirs obsédants, obsessionnels. Y songer encore n'en fera qu'en souligner la portée, voire en accroître les effets. Le temps n'y fait rien, n'atténue ni n'efface. L'horreur, pour les personnages d'Ewers qui un jour l'ont vécue et par la suite racontent, ne s'oublie pas. Telle est aussi la fonction de cette situation narrative initiale, même si - surtout si - elle installe cette distance temporelle entre les faits et leur narration : raconter encore revient à ressasser, revivre ad nauseam ce que l'on a vécu un jour.

Le récit enchâssé est donc pris en charge par un narrateur s'exprimant à la première personne, relatant sa propre histoire, témoin direct et privilégié des faits qui se sont produits. Le narrateur se mue, le temps que dure son récit, en autobiographe. Là non plus, Ewers n'innove pas. Le "phénomène" - quel qu'il soit - implique un être qui le perçoit ; il n' "existe" même qu'à travers cette conscience percevante, que par le truchement du témoin qui a "vu" "la chose". Et le "phénomène" sera d'autant plus "vraisemblable", "réel" qu'il aura été perçu par plusieurs témoins - c'est le cas dans cette nouvelle, on y reviendra -, et, dès lors, d'autant moins explicable par quelque hallucination, rêve ou autre folie d'un témoin qui serait unique.

Mise en action traditionnelle donc de la part d'Ewers dans Le Juif mort, mais qui n'en est pas moins remarquablement conduite.

Le récit enchâssé nous introduit dans le milieu des corporations d'étudiants, qu'Ewers connaissait bien, avec leurs habitudes, leurs rituels, leurs codes. Le narrateur doit assister à un duel opposant un étudiant en philosophie, juif, Selig Perlmutter, à un condisciple à l'évidence antisémite qui l'a insulté et s'en est pris à lui lors d'une altercation. Perlmutter ne s'est pas laissé faire, a répondu aux injures en giflant son adversaire, ce qui explique le duel. Le portrait physique de l'étudiant juif qui est brossé n'est assurément pas à l'avantage de ce dernier : personnage laid, misérable, pitoyable. De ce portrait on retiendra aussi - le détail a son importance - que le jeune homme souffre d'un très fort bégaiement. Le duel au pistolet se

10 “... Also bitte: erleichtern Sie sich", Ibid. 
déroule, selon un rituel bien précis. Avant qu'ils ne tirent est notamment prévue une tentative de réconciliation entre les adversaires. Mais au moment où Perlmutter souhaite s'exprimer, l'un des témoins, en toussant sciemment et violemment, l'empêche d'achever une phrase commencée, pour cause de bégaiement, avec bien des difficultés. Les spectateurs en concluent que Perlmutter refuse toute réconciliation. Après deux premières tentatives au cours desquelles il refuse de tirer, on l'enjoint de faire feu, il finit par obtempérer, manque son adversaire qui, lui, le tue d'une balle en plein front.

Du récit du narrateur jusqu'à cet instant, on retiendra l'histoire somme toute sordidement banale d'un duel entre des membres de corporations d'étudiants coutumiers du fait, le comportement pour le moins sujet à caution du témoin à l'égard de Perlmutter quand il ne le laisse pas s'exprimer (redoute-t-il une réconciliation entre les adversaires, ce qui mettrait fin d'emblée au "spectacle" que constitue aussi un duel ?), l'ignorance dans laquelle on demeure quant aux intentions de l'étudiant (qu'aurait-il dit si on l'avait laissé s'exprimer ?), son inertie pendant le duel (deux fois, il refuse de tirer : peur, lâcheté, indifférence, fatalisme ?).

L'histoire, dans sa phase suivante, tourne au macabre, ce qui ne surprend pas dans l'univers d'Ewers. Le cadavre doit être livré par ses compagnons au plus proche hôpital. Il est installé dans la diligence, sur un siège, aux côtés de ses accompagnateurs qui le retiennent chaque fois qu'il menace de basculer en avant à cause des soubresauts du véhicule. On boit pour tenter d'oublier l'encombrante présence. L'hôpital prévu, arguant de subtilités administratives, refuse de prendre en charge le corps. Il faut continuer le voyage vers un autre établissement. Cette fois le cadavre est attaché sur son siège avec une courroie et... les bretelles des voyageurs. Scène macabre et grotesque tout à la fois. L'atmosphère et le décor alentour sont à l'unisson : obscurité pesante, nuages bas, pluie battante qui détrempe le chemin, boue ou s'engluent les roues de la diligence et qui gicle sur les fenêtres, les opacifiant, air vicié et étouffant à l'intérieur du véhicule.

C'est dans ce décor, et préparé, progressivement amené ainsi, que va se produire - dernière phase de l'histoire, du récit du narrateur - l'inconcevable. Comme lors de la première étape, il s'agit cette fois encore de faire en sorte que le voyage se déroule au mieux en compagnie du cadavre qui - Ewers n'épargne, comme souvent, aucun détail - commence à "puer"11 ! On boit force bouteilles, on chante, on trinque à la santé du mort dans la bouche duquel on fait même couler du vin avant d'y glisser un cigare, on se met à jouer au skat, le mort, à qui l'on confie aussi des cartes, faisant le quatrième. Passe-temps qui tente aussi de dissiper le malaise ambiant, plaisanteries douteuses de carabins - l'un des voyageurs est étudiant en médecine et friand notamment d'histoires de dissection. Tout cela est sordide et macabre mais n'a en soi rien de terrifiant, nul fantastique n'est à l'œuvre ici, le mort se

11 "Ich glaube, er stinkt schon, sagte ich", $D G, 233$. 
borne à être un cadavre, non point un revenant, un spectre ou quelque autre figure de hantise. Mais les faits n'en restent pas là.

On pourra tout d'abord s'étonner qu'au skat c'est le mort qui gagne, partie après partie, comme s'il avait l'étrange pouvoir d'obtenir à chaque fois les meilleures cartes que l'un des trois autres joueurs nécessairement lui distribue ! Et ses compagnons de jeu ne manquent pas de lui glisser dans la poche l'argent ainsi gagné. On comprend ensuite l'étonnement, puis l'effroi, des voyageurs lorsqu'ils entendent le mort féliciter le narrateur à l'occasion de son anniversaire - c'est le fameux jour dont il a été question -, tout comme ils viennent de le faire eux-mêmes, puis trinquer à sa santé, reprendre une injure prononcée par l'un des personnages envers l'un des deux autres, rire lorsque ce même personnage le nargue et le provoque ; on comprend leur frayeur encore lorsqu'ils entendent une dernière fois ce rire "effroyable" après que l'homme lui a tiré une balle de pistolet en pleine tête. Ces faits ainsi présentés, l'épilogue du récit du narrateur sera laconique ; de ce qui s'est passé ensuite, il ne se souvient que "comme dans un brouillard"13. L'essentiel a été dit avant, il faut encore préciser - cela a son importance - que, le voyage achevé, l'auteur du coup de feu a été emporté "hurlant", "se débattant", "de la bave sur la bouche" ${ }^{, 4}$, qu'on lui a mis la camisole de force avant de l'enfermer dans l'asile où, quatorze ans après les événements, il se trouve encore. Les médecins expliqueront par l'alcoolisme ce qu'ils diagnostiqueront comme "un délire paranoïaque aigu"". On n'apprendra pas ce qu'il est advenu du cadavre, pas plus que l'on ne saura si les événements de la diligence ont été racontés à d'autres par ceux qui en ont été les témoins. Gageons que non. En revanche, comme on le voit dans l'ouverture du récitcadre, bien des années après, ils se plaisent (se complaisent ?) à les relater encore, à les écouter de nouveau.

Vengeance posthume de l'étudiant juif envers celui qui ne l'a pas laissé s'exprimer au moment du duel, tandis qu'il cherchait peut-être à se réconcilier avec son adversaire et qui, dès lors, indirectement, serait responsable de sa mort ? Explication possible et pensable. Victime condamnant à la folie celui qui, d'une certaine manière, fut son bourreau ? Interprétation plausible. C'est cependant moins à cette fonction potentielle du "phénomène" - rien dans le texte n'est explicite, et, par exemple, cette conclusion n'est nullement proposée par le narrateur et son interlocuteur qu'au "phénomène" lui-même qu'il convient ici de s'intéresser.

12 "Aber durch den Pulverdampf hindurch klang noch einmal das entsetzliche Lachen des Selig Perlmutter", $D G, 239$.

13 "- wie wir ankamen — das alles erinnere ich mich nur wie im Nebel ...", DG, 240.

14 "Ich hörte ihn schreien und brüllen, ich sah, wie er um sich schlug und wie ihm Schaum vor den Mund trat", Ibid.

15 "Akute Paranoia, hervorgerufen durch chronische Alkoholvergiftung, stellten die Ärzte fest", Ibid. 
On pourra toujours considérer l'épisode grotesque - comme "revenant", Perlmutter bégaie tout autant que lorsqu'il était vivant !-, il n'empêche, on a bien affaire à "quelque chose" qui est logiquement invraisemblable et pourtant incontestablement là, des plus perturbants, déstabilisant à l'extrême pour celui qui en est le témoin. "Quelque chose" advient, qui ne devrait pas se produire. Un "être" survient, qui ne devrait plus pouvoir le faire. Et Ewers prend bien soin de gommer les explications rationnelles qui pourraient être fournies et feraient que ce "phénomène" n'en est pas un. Outre le personnage directement victime, ses deux compagnons de voyage, dont le narrateur, "voient" et "entendent" eux aussi le mort. Ce qui pourrait être interprété comme l'hallucination d'un seul est beaucoup moins admissible quand il y a plusieurs témoins. L'hallucination collective n'est guère envisageable. La présence de plusieurs personnes, en revanche, accrédite l'inconcevable. Et l'explication un instant indirectement avancée par le témoin principal luimême - "vision" produite par la quantité d'alcool ingurgitée depuis le début du voyage - ne tient pas non plus : les "interventions" successives du mort, leur "cohérence" récusent d'emblée la thèse de l'alcoolisme chez les autres.

Voilà donc un mort qui réagit, rit, bégaie après son trépas, un mort qui paraît plus vivant même qu'il ne le fut lorsqu'il vivait, un spectre qui, insensible aux balles de pistolet, est immortel, revenu brusquement parmi les vivants, pour le plus grand malheur de ceux-ci qui, lorsqu'ils ne deviennent pas fous, ne peuvent s'empêcher de relater encore et encore, bien des années après, comme obsessionnellement, cette fatale rencontre.

\section{Les dernières volontés de Stanislawa d'Asp : Le rire de la momie}

Aucun narrateur s'exprimant à la première personne ne prend ici en charge le récit qui est le fait d'un conteur omniscient nous faisant entrer de plain-pied dans l'histoire. Le premier tiers de la nouvelle relate des passions amoureuses. Celle tout d'abord qu'éprouve le comte Vincenz d'Ault-Onival à l'égard de Stanislawa d'Asp - de son vrai nom Lea Lévi -, une chanteuse de cabaret débauchée et phtisique, mi-artiste, mi-courtisane, qui ne manque jamais d'humilier le comte. Vincenz la fait soigner et, séduite par un tel amour qui force son respect et son admiration, Stanislawa finit, elle, la juive, par accepter d'épouser le fervent catholique qu'il est, alors que "ce n'était pas lui qu'elle aimait, mais uniquement son immense amour" "La seconde passion est celle que va éprouver Stanislawa cette fois pour Jan Olieslagers, ami d'enfance du comte, venu rendre visite au couple. Le Flamand représente pour Stanislawa, dans la vie bien ordonnée qu'elle mène aux côtés de Vincenz, l'originalité, le changement, le mouvement. Grand voyageur, sans

\footnotetext{
16 "Und als sie dann anfing zu lieben — und als sie liebte — li e b t e s i e d o c h n i c h t
} i h n, s o n d e r n n u r s e i n e gro $\beta$ e L i e b e", $D B, 14$. C'est Ewers qui souligne. 
attaches, qui "a l'habitude de prendre ce qui lui plaît"17, Jan, quant à lui, devient l'amant de Stanislawa mais ne tarde pas à annoncer, après quelques semaines passées au château, qu'il souhaite s'en aller de nouveau parcourir le monde. Pensant que l'amour de Vincenz pour elle ira jusqu'à lui faire accepter le sacrifice extrême qu'elle exige de lui, Stanislawa demande au comte de lui donner l'argent qui lui permettra d'accompagner son amant. Vincenz refuse.

Amours croisées, êtres "possédés" par leur passion, femme séductrice qui peut être aussi séduite, aventurier sans états d'âmes, violence des sentiments et des comportements : la nouvelle, en ses treize premières pages, déroule semblable intrigue et pourrait se clore, pour Stanislawa, sur une séparation et une vie de résignation. Mais la jeune femme va organiser pour son mari et son amant de machiavéliques et macabres retrouvailles.

Au bout d'un an et demi, Jan reçoit une lettre du comte lui annonçant la mort de sa femme. Une seconde lettre, un an plus tard, le prie de revenir au château pour accomplir "les dernières volontés" de la comtesse. Stanislawa, avant de mourir, a demandé au comte qu'au bout de quelques années dans la tombe ses restes soient entreposés dans une urne dans la chapelle du château. Il n'y a là rien de surprenant, cela correspond à une vieille tradition familiale, tous les ancêtres du comte ont ainsi leur place dans la chapelle. Plus étonnant est en revanche le serment que la jeune femme exige de Vincenz sur son lit de mort, celui d'exécuter scrupuleusement ses "dernières volontés", dont elle a elle-même fixé avec précision le déroulement, lorsque le jour du transfert des restes sera venu, tout devant se faire un après-midi ensoleillé, en présence de Jan, et être achevé avant le coucher du soleil. Autre détail singulier : pour donner plus de pouvoir au serment qu'elle impose à son mari, avant de mourir, elle se convertit au catholicisme, le catholique fervent qu'est le comte pourra ainsi d'autant moins se défaire de ce qu'il aura juré sur la croix qu'elle tient entre ses mains au moment où elle exige cette promesse de Vincenz. Énigmatiques, inquiétantes aussi sont ses dernières paroles et le sourire que ses lèvres conserveront après qu'elle aura rendu le dernier souffle, lorsque le prêtre qui l'assiste lui parle de résurrection et de retrouvailles après la mort : "Oui mon père [dit-elle à propos de son mari], vous pouvez le croire : il me reverra certainement $"$ "18.

De la vie de sa femme entre le départ de Jan et sa mort, le comte n'a que peu de choses à relater, sinon qu'elle paraît ne plus attacher d'importance à l'existence tout en soignant avec une extrême attention son corps, à l'aide de mystérieux produits qu'elle se procure, "une pâte brillante" en particulier, "un vernis à porcelaine" ${ }^{19}$ qu'elle fait venir de Prague, dont, durant des mois, elle

17 "Dem Vlamen gefiel diese Frau und er war es gewohnt, das zu nehmen, was ihm gefiel", $D B, 18-19$

18 “Ja, Pfarrer, das dürfen Sie glauben: mich wird er gewiss wiedersehen", DB, 31. C'est Ewers qui souligne.

19 “... das andere, das aus Prag kam, eine glänzende Pasta, die sich 'Porzellanschminke' nannte", $D B, 35$ 
s'enduit le visage, étrange fard qui permet à ses traits de conserver leur ancienne beauté, ainsi que le montre à l'envi le portrait fait d'elle sur son catafalque.

Le dernier tiers de la nouvelle fournit les ultimes explications à ces agissements aussi étranges qu'inquiétants, et l'on ne peut qu'être sensible à la construction rigoureuse du récit, à l'art avec lequel Ewers fait monter la tension, ménage ses effets, paraît dévoiler des éléments pour, en réalité, mieux dissimuler encore et alourdir l'atmosphère. La manifestation du "phénomène" demande ce travail précis de préparation, elle sera d'autant plus impressionnante qu'elle aura été amenée de la sorte, annoncée, mais sans que, jamais, le lecteur, lorsqu'il découvre la nouvelle pour la première fois, ne puisse savoir en fait ce dont il s'agit.

Le jour où toutes les conditions requises pour le transfert des restes sont réalisées, lorsque le comte en compagnie de Jan et de trois jardiniers - la présence de témoins est importante - fait ouvrir la tombe et le cercueil, il découvre le corps de Stanislawa intact, exactement semblable à celui qui, trois ans plus tôt, était allongé sur le catafalque ; sur les lèvres, légèrement colorées de rouge, le même sourire énigmatique. Lorsque Jan, descendu dans la tombe, tapote la joue de la morte, "un son léger très délicat" se produit, "comme s'il touchait une porcelaine de vieux Sèvres"20. Dès lors, les éléments précédemment distillés dans le récit se rejoignent, l'on déchiffre l'énigme, la perspective qui se dessine est dans cette nouvelle aussi sordide et macabre : le cadavre de Stanislawa ne s'est pas décomposé avec le temps (c'est l'effet des produits utilisés par la comtesse). Comment alors placer les restes dans l'urne si ce n'est en découpant, dépeçant le cadavre, tâche à laquelle devra s'astreindre - serment oblige - le comte, et ce le jour même, avant le coucher du soleil. Vincenz "revoit" en effet son épouse après la mort, comme Stanislawa le lui avait promis, mais de quelle manière, en quelles circonstances ! Après avoir compris ce dont il s'agit réellement, le comte demande tout d'abord au plus vieux des jardiniers de découper le corps, ce qu'il refuse, l'un des deux autres, plus jeune, finit par accepter mais se récuse aussitôt et s'enfuit, terrorisé, ayant eu l'impression que le cadavre - que Stanislawa - "se moquait" de lui ${ }^{21}$. Sollicité par Vincenz, Jan refuse de la même manière. C'est au comte, et à personne d'autre, de s'acquitter de cette tâche, conformément au serment qu'il a fait. Il s'exécute donc, accompagné du sourire de la comtesse, tandis que lui se met à rire, d'un rire "de dément" ${ }^{, 2}$. Les jardiniers quittent précipitamment les lieux, Jan s'éloigne lui aussi, et la fin de l'épisode est évoquée à partir de la perspective de ce témoin visuel et surtout auditif ; point de vue essentiel car il accrédite l'ensemble et la manifestation du "phénomène" en particulier. Car avant d'apercevoir dernières lignes de la nouvelle - le comte se diriger, un rictus sur le visage,

20 "Er beugte sich tief nieder und knipste leicht mit dem Nagel auf der Toten Wange. Es gab einen ganz feinen leisen Ton, als ob er altes Sèvres berühre", $D B, 40$.

21 "Nein, nein! schrie er. Sie lacht mich aus", $D B, 40$. C'est Ewers qui souligne.

22 “... das wahnsinnige Gelächter des Grafen”, $D B, 46$. 
une urne à la main, vers la chapelle, tandis que le soleil se couche à l'horizon - "dernière volontés" de la comtesse en effet accomplies -, il aura entendu lui aussi "un son clair, argentin, comme provenant des lèvres d'une femme", 23 , "de plus en plus fréquent, de plus en plus distinct", "des sons bestiaux"24.

Il convient, bien entendu, à l'instar de ce que fait Jean-Jacques Pollet, de replacer cet épisode final dans l'économie générale de la nouvelle ${ }^{25}$. Il s'agit bien de la vengeance machiavélique, perverse et cruelle de Stanislawa à l'endroit de son mari, mais aussi de son amant, convié par elle à l'exécution de ses dernières volontés. Sa mort "physique" avait déjà privé Vincenz de l'être le plus cher à ses yeux, de la femme à qui il vouait depuis toujours une passion dévorante. En le forçant - et de quelle manière ! - à détruire luimême, après sa mort, son corps, en respectant, qui plus est, une tradition ancestrale dans la famille du comte, elle poursuit et parachève une vengeance démoniaque. En imaginant, trois ans auparavant, que l'amour de son mari serait à ce point absolu qu'il lui aurait - aussi - permis de la laisser partir avec son amant, elle s'est trompée. L'amour de Vincenz est en effet absolu et le comte ne saurait donc tolérer qu'elle le quitte. La vengeance de la comtesse sera, alors, elle aussi extrême. Stanislawa s'est en quelque sorte momifiée vivante. Par des produits appropriés, elle parvient à figer son corps, à le pétrifier, de manière à ce que, devenu cadavre, il ne subisse pas les effets de la décomposition. Si un tel procédé, qui arrête le cycle biologique, pouvait surprendre à l'époque d'Ewers - encore qu'il s'agisse aussi du rêve éternel de l'homme souhaitant arrêter le temps, en annihiler les effets dévastateurs, mythe intemporel d'accession à l'"éternité -, peut-être étonnerait-t-il moins de nos jours ${ }^{26}$, sera-t-il même maitrisable à l'avenir. Ce à quoi se livre Stanislawa sur son corps ne relève pas du domaine strict de l'impossible, du scientifiquement inconcevable et inexplicable, donc pas du fantastique. Même si elle n'est sans doute pas à l'époque d'Ewers expérimentalement vérifiable, l'explication rationnelle et scientifique pourrait être avancée.

Le fantastique naît ici - simplement, serait-on tenté de dire, mais tout, précisément, est dans ce détail ténu du texte - dans le sourire du cadavre, dans le fait que le premier jardinier commis au "découpage" a le sentiment que Stanislawa se moque de lui - impression, certes, mais tout tient à cette impression, qui plus est d'un personnage extérieur au trio -, puis dans le rire, plusieurs fois évoqué, qualifié de façon différente, et entendu par cet autre témoin de la scène qu'est Jan - importance décisive de l'autre personne qui,

23 "Aber dazwischen - ein anderer Ton. Leicht, silberhell, wie von Frauenlippen. Was war es nur?", Ibid.

24 "Es kam wieder und wieder — öfter und deutlicher — was war es nur?

Und dann, plötzlich wusste er es: die Gräfin lachte. [...] So hockte er da, wie ein angeschossenes Wild, wagte es nicht, diese tierischen Töne zu lassen ...", $D B, 46-47$. C'est Ewers qui souligne.

25 Jean-Jacques POLLET, Essai sur la littérature fantastique allemande du début du XX siècle, p. 257-258.

26 Voir, par exemple, les travaux actuels sur la cryogénisation, même s'ils sont aussi perçus avec scepticisme par les scientifiques. 
elle aussi, perçoit le "phénomène". Ces "réactions" du cadavre intact dans son cercueil font de lui un spectre, le mort est aussi, encore, un vivant, revenu parmi les vivants - les a-t-il jamais quittés ? -, pour leur plus grand malheur. On ne sait pas vraiment si Vincenz est devenu fou, son rire est qualifié de "dément" certes, mais c'est une impression de Jan et le texte ne sera pas plus explicite. Mais peu importe en définitive. Le spectre aura conduit le mortel sur lequel s'est concentrée sa haine très exactement où il voulait l'amener, il l'aura forcé à accomplir très exactement ce qu'il souhaitait qu'il fasse. Et fondamentalement pour l'approche ici choisie dans l'analyse : le spectre se sera manifesté, l'objet de terreur se sera imposé, en dépit de son impossibilité théorique, c'est-à-dire rationnelle.

\section{L'Araignée : Femme-araignée fatale}

La structure de la nouvelle L'Araignée diffère encore de celle des deux autres œuvres. Les cinq premières pages - récit pris en charge par un narrateur omniscient - relatent des faits. Les vingt-huit pages suivantes sont constituées par le Journal qu'a tenu, vingt et un jours durant, l'un des personnages évoqués dans la première phase de la nouvelle, "l'étudiant en médecine Richard Bracquemont", récit à la première personne qui s'achève sur les derniers mots rédigés par le scripteur juste avant qu'il ne se suicide. La dernière page met en scène un commissaire qui trouve et lit le Journal, la réaction qu'il a alors donne son véritable sens à la nouvelle. Ultime signification tout entière contenue dans la phrase par laquelle se clôt 1 'ensemble ${ }^{27}$.

La nouvelle, en sa première phase, commence comme une intrigue policière. Dans une chambre d'un petit hôtel parisien, trois personnes se pendent à la crémone de la fenêtre, le vendredi, trois semaines de suite. Rien ne prédisposait aucune d'entre elles à se suicider, et notamment pas la troisième, un agent de police aguerri et peu impressionnable, mandaté par le commissaire de l'arrondissement pour tenter de percer le mystère des deux premiers suicides. Arrivé dans la chambre le dimanche soir, le policier fait chaque jour son rapport, le mercredi il prétend être sur une piste sans vouloir trop en dire pourtant, le vendredi il évoque l'étrange fascination que parait exercer sur lui la fenêtre de la chambre ; le soir on le retrouve mort. Dans cette relation a priori objective de faits incontestables, certains éléments, d'emblée, installent cependant l'étrangeté, sans que l'on puisse en percevoir

27 Wilfried Kugel, dans sa biographie d'Ewers (Der Unverantwortliche. Das Leben des Hanns Heinz Ewers, Düsseldorf, Grupello Verlag, 1992), voit des analogies possibles entre la nouvelle d'EWERS et celle publiée par ERCKMANN-CHATRIAN, plus de quarante ans auparavant, en 1862, dans les Contes $d u$ bord du Rhin, "L'œil invisible". Certains rapprochements sont certes possibles, mais EWERS écrit un récit radicalement différent de celui des deux auteurs français, infiniment plus étrange. On pourra lire la nouvelle d'ERCKMANN-CHATRIAN in : L'œil invisible. Contes fantastiques, t. 2, Talence, Editions de l'Arbre vengeur, 2008. 
les tenants et aboutissants : les trois suicides dans la même pièce, le même jour de la semaine, le rôle joué par la fenêtre, une araignée aussi - première illustration du titre - qui sort de la bouche du policier lorsqu'on examine son corps, ou que l'on aurait vu courir sur l'épaule du premier suicidé.

Le cadre ainsi posé, le Journal rédigé par Richard va ensuite dérouler les faits dans toute leur inexorabilité. Subterfuge par lequel le jeune homme a obtenu la chambre - il a prétendu auprès du commissaire pouvoir résoudre l'énigme -, agents de police patrouillant dans la rue de l'hôtel prêts à intervenir au moindre signe de l'occupant de la chambre, ligne téléphonique directe entre la pièce et le commissariat, mise à la disposition de l'étudiant d'un revolver, récit des événements de la première semaine, le fameux vendredi inclus, où rien ne se passe : ainsi se résument les premières notations du diariste ; le récit policier se poursuit, l'enquêteur est à l'œuvre et ses investigations ne donnent rien, même s'il précise aussi éprouver parfois une certaine envie d'aller à la fenêtre. Pourtant il ne songe pas à quitter la chambre, une "raison", en effet, le retient en cet endroit ${ }^{28}$ : une jeune femme, qu'il nomme Clarimonde ${ }^{29}$, aperçue dès les premiers jours de l'autre côté de la rue, assise à une fenêtre donnant juste sur la sienne et l'observant. Cet élément précisé, qui complète et explique la première allusion à la fenêtre, l'engrenage se met en place dont Richard ne se défera plus. Des relations ne tardent pas à s'instaurer entre les deux voisins qui, commencées sous la forme de ce qui peut paraître un jeu, vont se révéler fatales pour l'étudiant. Des sourires sont échangés, puis des signes de la main, les regards sont de plus en plus appuyés, Richard se met à faire des gestes que la jeune femme imite à l'identique et à la seconde près où ils sont effectués par l'étudiant, jeu étrange qui se poursuit, se précise, s'accentue des jours durant, finit par occuper entièrement Richard à tel point que certaines notations de son Journal se bornent à mentionner que, tel jour, Clarimonde et lui "ont joué ensemble toute la journée", jusqu'à ce qu'il finisse par s'apercevoir que ce n'est pas lui qui joue avec elle, mais elle avec lui ${ }^{30}$, qu'il est entièrement sous l'empire de sa mystérieuse voisine et qu'elle lui impose ses volontés. Lorsque, le vendredi 25 mars - le fameux et fatidique jour de la semaine -, chez elle, elle détache le cordon de ses rideaux, y fait un nœud coulant et le fixe au crochet de la crémone, dans sa chambre, l'étudiant reproduit le geste ; le soir même, le

28 "Freilich kann ich nicht leugnen, dass ich manchmal einen gewissen Drang verspürte, zum Fenster zu gehen - o ja, aber aus anderen Gründen! [...] Und endlich habe ich noch einen Grund, der mich hier hält", $D B, 97-98$.

29 "Clarimonde" est le prénom du personnage féminin de la nouvelle de Théophile Gautier, La Morte amoureuse (Ewers, on le sait, a traduit des œuvres de l'auteur français), courtisane qui survit après la mort, aimée d'un jeune prêtre ; personnage en définitive moins maléfique que son homologue chez Ewers, même si elle boit le sang du prêtre pendant son sommeil, juste ce qui convient à sa "survie" toutefois ...

30 "Sonntag, 20. März.

Ich kann heute nur wieder schreiben: den ganzen Tag haben wir gespielt”, $D B, 113$.

"Ich habe eine Entdeckung gemacht: ich spiele nicht mit Clarimonde - sie spielt mit mir", $D B, 115$. C'est Richard qui souligne. 
commissaire découvre le corps de Richard pendu à la fenêtre, exactement dans la même position que les trois suicidés précédents.

Outre les faits, le Journal relate aussi, jour après jour, les sentiments éprouvés, impressions ressenties, interrogations émises, hypothèses formulées par celui qui est le témoin et, finalement, la victime des agissements de la mystérieuse dame à la fenêtre. Le procédé narratif choisi par Ewers pour cette nouvelle est particulièrement judicieux : le diariste relate ainsi, quasiment en temps réel, les événements auxquels il assiste et la manière dont il les vit. Et le dernier jour, à l'ultime instant, avant qu'il ne cède à l'irrésistible tentation de se rendre à la fenêtre, sachant qu'il ne pourra faire autrement que de s'y rendre et qu'il le fera pour se pendre, le temps de l'écriture rejoint le temps de l'événement vécu, dans une coïncidence parfaite : Richard ne parvient plus à écrire autre chose que son nom, puis son nom incomplet ${ }^{31}$. Point final du Journal, écriture interrompue, vie achevée. Simultanéité du mot écrit et de l'acte accompli. On peut même dire que si cette histoire est celle d'une dépossession, le Journal de Richard qui, par l'acte d'écriture même qu'il implique, devrait permettre au jeune homme de "se retrouver", exprimera au contraire, jusqu'à la dernière seconde, cette dépossession, le diariste ne parvenant même plus à écrire en entier son nom ; on ne saurait mieux dire cette dissolution de l'identité.

Richard ne dissimule rien de ce qu'il ressent au fur et à mesure que passent les jours en compagnie de l'étrange et inquiétante voisine. Fonction et vertu du Journal encore que de permettre cette introspection précise. Impression que la jeune femme, d'emblée, dès qu'ils se sont aperçus mutuellement à la fenêtre, s'intéresse à lui, qu'il lui plaît, que ses pensées à lui sont de plus en plus accaparées par la présence et la personnalité de Clarimonde, incapacité croissante de travailler, de se plonger dans ses livres, de se concentrer sur ses études, envie de plus en plus irrépressible de se rendre à la fenêtre, d'y rester, fascination grandissante exercée par ce lieu, interrogations sur ce qu'il éprouve réellement pour Clarimonde (est-ce de l'amour ?), jusqu'à ce qu'il finisse par admettre que c'est bien de cela, en apparence, qu'il s'agit, mais aussi inquiétude, angoisse, "sensation tyrannique et singulièrement voluptueuse" ${ }^{32}$ devant quelque chose qu'il ne connaît pas, certitude encore qu'il lui faudra à un moment aller vers elle, peur qu'il lui arrive, à lui aussi, ce qui est advenu à ses prédécesseurs, terreur lorsqu'il s'imagine, lui aussi, pendu au bout d'une corde, volonté de mettre un terme à tout cela en prévenant le commissaire et incapacité à avouer à ce dernier ce qui se passe réellement, tentative - timide - pour se distraire (il accepte, sans conviction, l'invitation à dîner du policier), décision de s'isoler entièrement

31 "Meinen Namen — Richard Bracquemont, Richard Bracquemont, Richard — o, ich kann nicht mehr weiter - Richard Bracquemont - Richard Bracquemont — jetzt — jetzt, ich muss sie anschauen - Richard Bracquemont — ich muss — nein, noch mehr — Richard Richard Bracque ——, $D B, 121$.

32 "Und gerade diese Angst ist es, die etwas seltsam Bezwingendes, merkwürdig Wollüstiges hat, die mich von ihr abhält und doch näher zu ihr hinzieht", $D B, 109$. 
du monde pour ne plus vivre que dans sa chambre et à cette fenêtre (après avoir vu Clarimonde faire de même chez elle avec un téléphone, il coupe le fil de l'appareil le reliant au commissariat), ultime réaction de résistance avant de céder à la tentation suprême, d'exécuter le geste fatal.

Richard écrit ainsi la chronique d'un envoûtement, d'un asservissement progressif, celle d'un suicide annoncé. Fascination irrépressible à laquelle on succombe ? Assurément. Jeu aussi pervers que voluptueux auquel on se livre ? A n'en pas douter. Sentiment de jubilation éprouvé à l'idée même que l'on ne pourra résister, quand bien même la mort serait-elle au rendez-vous ? Tout autant. "Désir et désir de mort", "femme fatale, figure clé du décadentisme" à laquelle on s'abandonne "avec volupté" ? On peut en effet suivre Jean-Jacques Pollet encore dans cette interprétation ${ }^{33}$.

Mais il y a plus, et plus étrange.

Car, dans son Journal, Richard brosse aussi un portrait physique de Clarimonde. Cheveux noirs et ondulés, teint et lèvres pâles, de petites dents qui paraissent à l'étudiant "aussi aiguisées que celles de bêtes sauvages ${ }^{34 ،,}$, de grands yeux sombres qui brillent sous de lourdes paupières : quelque mystère, à tout le moins, se dégage de cette évocation, la comparaison avec les animaux de proie n'étant pas l'un des éléments les moins inquiétants. Précisons encore la tenue de la jeune femme, toujours identique : un fourreau noir, semé de grandes taches couleur lilas, de longs gants noirs qui rendent ses doigts, lorsqu'ils s'affairent, semblables à des "pattes d'insectes",35. Inquiétante apparition décidément. Ajoutons l'activité à laquelle Clarimonde se livre, lorsqu'elle n'imite pas les gestes effectués par son voisin : elle file avec une quenouille ancienne dont elle dévide "des fils incroyablement fins ${ }^{\prime 36}$, travail qu'elle effectue inlassablement la journée entière jusqu'à la nuit tombante, à la fenêtre de cet appartement qu'elle paraît ne jamais quitter. Clarimonde la fileuse à la quenouille, à l'image de la Parque Clotho qui tenait le rouet, et qui devient Atropos l'inflexible - celle qui coupait le fil des destinées humaines -, lorsqu'elle attire Richard vers le cordon fatal - autre fil - auquel il se pendra : le rapprochement est aisé à faire. Ajoutons que les fils "incroyablement fins" que Clarimonde sait tirer de l'instrument rappellent les fils avec lesquels l'araignée - dont il a été question lorsque l'on a retrouvé l'arachnide sur les cadavres de deux des suicidés précédents - tisse sa toile.

33 Jean-Jacques POLLET, Introduction à la nouvelle fantastique allemande, p. 68. Sur le personnage de la "femme fatale" (en France, Angleterre et Italie), voir aussi : Mario PRAZ, La chair, la mort et le diable dans la littérature du XIX siècle. Le romantisme noir. Traduit de l'italien par Constance THOMPSON PASQUALI, Paris, Editions Denoël, 1977. Voir en particulier le chapitre 4 (p. 163-243), "La belle dame sans merci".

34 “... und es scheint mir, als ob die kleinen Zähne zugespitzt wären wie bei Raubtieren”, $D B, 100$.

35 "Es sieht seltsam aus, wie die schmalen schwarzen Finger, schnell, scheinbar durcheinander, die Fäden nehmen und ziehn - wirklich, beinahe wie ein Gekrabbele von Insektenbeinen", $D B, 100-101$.

36 “... es müssen ungeheuer zarte Fäden sein, die sie macht”, $D B, 100$. 
Cet animal est une nouvelle fois évoqué au cours d'un épisode que Richard relate aussi, assez longuement - près de deux pages -, dans son Journal : le spectacle auquel il a assisté dans le couloir menant à sa chambre, celui d'une araignée - "une grosse aranéide crucifère" "37 - attirant un mâle au milieu de sa toile, l'étreignant, le poursuivant ensuite tandis qu'il tente de s'échapper, avant de le tuer en aspirant "le jeune sang de son amant à longs traits" et de rejeter ensuite hors de sa toile "le petit tas pitoyable et informe pattes, peau et fils ${ }^{\prime 38}$. Scène courante de la vie animale montrant l'araignée qui, à l'instar de la mante religieuse, tue et dévore - et de quelle manière ! le mâle après s'être accouplée avec lui, mais scène observée puis contée avec un luxe de détails par Richard, aussi horrifié que fasciné par le spectacle. Dernière évocation de l'animal dans la nouvelle : lorsque le commissaire découvre le cadavre de Richard, il remarque entre ses dents, "broyée et écrasée, une grosse araignée noire, avec d'étranges taches violettes" ${ }^{\text {"39. }}$.

Que conclure?

On pourra penser qu'Ewers force le trait, qu'il livre (trop aisément ?) nombre d'éléments permettant le rapprochement : les cheveux noirs de Clarimonde et l'araignée noire trouvée sur les cadavres, les dents de la jeune femme assimilées à celles d'un animal de proie, les taches lilas sur son fourreau et les taches violettes sur l'araignée entre les dents de Richard, les longs gants noirs qui font des doigts "des pattes d'insectes", la fileuse à sa fenêtre et l'araignée - femelle - qui a tissé sa toile, l'arachnide qui tue le mâle après l'amour et Clarimonde poussant Richard, qui se dit amoureux d'elle, au suicide.

Il n'empêche : on se perd en conjectures quant à une interprétation possible, et l'auteur a le don de nous conduire, et jusqu'aux dernières lignes de la nouvelle, pour mieux nous laisser seuls et perplexes face à un sens à envisager. Bien sûr, il est loisible de voir symboliquement en Clarimonde l'incarnation de la femme fatale, femme sphinge énigmatique et inaccessible, cruelle et impitoyable, de la femme vampire, de la femme mangeuse d'hommes, dévorant ses amants, pour filer - si l'on ose dire - la métaphore...

On peut préférer à cette perspective une lecture littérale de la nouvelle où la dimension fantastique se révèle à plein ${ }^{40}$, sans que l'on soit pour autant à même de suggérer une interprétation définitive. Proposons donc, sous forme de questions. L'araignée a-t-elle pris un temps - celui de conduire au suicide successivement quatre personnes, quatre hommes - les traits d'une femme à laquelle on ne saurait résister, reproduisant sur son apparence physique

37 “... eine große Kreuzspinne”, $D B, 104$.

38 "Dann schlug sie die scharfen Zangen in seinen Leib und sog in vollen Zügen das junge Blut des Geliebten. Ich sah noch, wie sie endlich das jämmerliche, unkenntliche Klümpchen Beinchen, Haut und Fäden - loslöste und verächtlich hinauswarf aus dem Netz", DB, 105.

39 "Und zwischen ihnen [den Zähnen] klebte, zerbissen und zerquetscht, eine große schwarze Spinne, mit merkwürdigen violetten Tupfen”, $D B, 105$.

$40 \mathrm{Du}$ reste, tout fantastique n'implique-t-il pas cette lecture littérale au cours de laquelle nous croyons ce qui nous est dit, sans chercher nécessairement une fonction ou une raison d'être? 
nombre d'aspects qui caractérisent l'animal ? Capacité de l'animal à se métamorphoser en être humain ? Ou, à l'inverse, la mystérieuse femme à la fenêtre se mue-t-elle, une fois son forfait accompli, en cet arachnide qui porterait dès lors sur son corps les fameuses taches de couleur, venue retrouver une dernière fois les cadavres de ses victimes, jusqu'à finir broyée entre les dents du dernier suicidé ? Aptitude de l'être humain à se changer en animal?

On ne peut qu'en rester à ces hypothèses. Mais, au fond, l'essentiel est ailleurs : la nouvelle montre la confrontation de l'individu avec le "phénomène", quel qu'il soit, incompréhensible, inexpliqué, inexplicable (?), perturbant, mortel ; on est bien ici au cœur du fantastique ; peu importe la nature de la métamorphose, c'est la métamorphose qui prévaut, et ses conséquences.

Le fait qu'avant Richard trois autres personnes - trois autres hommes aient été victimes de "la chose" montre qu'il ne s'agit pas d'une hallucination de l'étudiant, ce qui détruirait d'office le fantastique. Et la dernière phrase du récit - ultime élément fourni par Ewers, magistrale coda de la nouvelle décrivant le commissaire se rendant, après avoir lu le Journal de Richard, dans la maison d'en face pour constater que le deuxième étage est vide depuis des mois et inoccupé, accentue encore le mystère ${ }^{41}$. Si l'appartement en face de l'hôtel est ainsi inhabité depuis longtemps, qu'ont bien pu y apercevoir Richard et les trois locataires précédents de la chambre ? De qui ou de quoi ont-ils bien pu être les victimes ?

On le voit, Ewers distille, au cours de son récit, avec un art consommé de la construction dramatique, maints éléments dont chacun, à sa manière, paraît apporter une précision, mais, en réalité, contribue à épaissir le mystère. Plus le récit se déroule, moins l'on comprend. Le fantastique, dans cette nouvelle, naît de ces interrogations et indécisions permanentes et qui demeurent jusqu'à la fin.

\section{Pluridimensionnalité}

Mort revenu de chez les morts au rire effrayant qui rend fou, morte au corps toujours intact dont le rire tout aussi effroyable rythme l'exécution des dernières volontés imposées, femme-animal fatale : les spectres se portent bien dans l'univers d'Ewers, que le nouvelliste montre, mette en scène ces objets de terreur avec la violence expressive qui leur sied (deux premières nouvelles), ou qu'il joue avec la plus grande habileté sur l'équivoque (troisième récit analysé). Les protagonistes de ces histoires, témoins et victimes du "phénomène", doivent brusquement faire face à un monde autre,

41 "Der Kommissar las es [das Tagebuch] und begab sich sofort in das gegenüberliegende Haus. Er stellte dort fest, dass der zweite Stock seit Monaten leer stand und unbewohnt war - ", $D B, 122$. 
à une "surnature" d'emblée en position dominante. A l'instar des autres auteurs fantastiques, Ewers met en place dans ces récits un arrière-plan présenté comme cohérent - une histoire de duel, des passions amoureuses, une manière d'enquête policière - sur lequel va venir s'inscrire avec d'autant plus de virulence "l'inquiétante étrangeté". Dès lors des notions considérées comme intangibles sont bouleversées, des cadres présentés comme établis transgressés, des frontières habituellement fixées effacées. La puissance subversive du fantastique fracture l'univers de référence qui est le nôtre et que nous considérons comme seul possible. Le fantastique est bien chez Ewers aussi émergence dans le quotidien ordinaire d'une réalité extraordinaire, surgissement dans le monde des humains d'un univers tératologique.

En ce début de siècle inscrit dans la continuité de celui qui s'achève, entre un modèle scientifique omniprésent et une religion dont les propositions ne sont plus satisfaisantes, voire plus crédibles, entre une raison érigée en principe suprême et un dieu vacillant, sinon occulté, le fantastique ewersien fait surgir un univers qui ouvre sur un au-delà ou un en-deçà possible du réel, complète et prolonge une réalité ordinairement admise d'une autre dimension communément refusée. 